\title{
Figuras de la muerte en la Fenomenología del espíritu*
}

\author{
Víctor Duplancic ${ }^{* *}$
}

\begin{abstract}
Resumen
El presente artículo ofrece una lectura transversal de la Fenomenología del espíritu de Hegel de la mano de la idea de muerte. Para ello se utilizan tres claves de lectura para mostrar las distintas figuras que la muerte asume en el texto. Estas tres claves son: a) la muerte como la culminación natural de la vida; b) el miedo a la muerte como idea regulativa en el proceso de constitución de una sociedad; c) la superación de la muerte mediante un modo racional-especulativo de sobrevivencia.

Palabras claves: Hegel, fenomenología del espíritu, muerte.
\end{abstract}

\section{Abstract \\ Figures of Death in 'Phenomenology of the Spirit'}

This paper proposes a transversal lecture of the Phenomenology of Mind from Hegel by following in the text the idea of death. To show the different figures of the death in the book, the author uses three lecture's key: a) the death as natural end of the life, b) the fear of the death as regulative idea by the constitution the society, c) overcoming death through one kind of rational way of survival.

Keywords: Hegel, Phenomenology of Mind, Death.

\footnotetext{
* Recibido: mayo 2017. Aceptado: julio 2017.

*** Universidad Nacional de Cuyo. Mendoza, Argentina. Email: victorduplancic@gmail.com
} 


\section{La idea de muerte en la Fenomenología del espíritu}

Un antecedente importante en el tratamiento de la muerte en la Fenomenología es sin duda la obra de Kojève (Kojève, 1947, 1972). Para él, como dice Bataille, la "idea central y última de la filosofía hegeliana", sería "la idea de que el fundamento y origen de la realidad objetiva (Wirklichkeit) y de la existencia empírica (Dasein) humanas son la Nada que se manifiesta en tanto Acción negativa y creadora, libre y consciente de sí misma" (Bataille \& Strauss, 1990; Gemerchak, 2012). Filosofía dialéctica sería así sinónimo para Kojève de una filosofía de la muerte. Ahora bien, no se puede decir tan fácilmente que la filosofía de Hegel, sobre todo el texto de la Fenomenología que tenemos por análisis en éstas líneas, es filosofía dialéctica como una filosofía de la muerte. La muerte está presente, como lo veremos, pero en figuras del camino hacia el saber absoluto que deben ser superadas por la vida del espíritu y cuya figura determinante epistemológicamente es la de muerte especulativa o de una muerte que es "contenida" dialécticamente en la vida del espíritu. Es también verdad que la fuerza negativa de la muerte es parte esencial de la dialéctica, pero la dialéctica no se reduce a ello, es más bien el método o la estructura misma de todo el desarrollo que va desde la conciencia inmediata o natural hasta el saber absoluto, y en esa estructura el momento negativo es factor constitutivo pero no determinante absoluto. Por ello mismo y por el hecho de que la interpretación de Kojève sería lo que podríamos llamar una lectura "externa" a la Fenomenología que tendría por objeto el encontrar en la idea de muerte el momento dialéctico para revertir una historia de servidumbre (reversión basada en una determinada idea de autonomía), hacen que haya decidido re-orientar mi lectura hacia un contexto más inmanente a la Fenomenología misma y a su realidad histórica propia. Por supuesto que esto no va en detrimento de la gran validez interpretativa de Kojève, es sólo una opción hermenéutica que tomo y que ciertamente no es en desmedro de la figura de la idea de muerte en la Fenomenología. Sólo que quisiera relativizar el hecho de que la muerte pueda ser que "la" clave de lectura. Es ciertamente una clave de lectura al modo de otros muchos conceptos fundamentales de la Fenomenología. Lo propio de estos conceptos es que experimentan transformaciones a lo largo de su recorrido, y esas transformaciones acompañan la historia inherente al texto mismo. Lo mismo sucede con la ida de la muerte. Tampoco quiero con ello descartar la validez de las figuras de la historia del mundo que se presentan en el texto y que dan el sustento legítimo para "salir" del texto y realizar interpretaciones históricas de términos usados por Hegel. Un ejemplo claro de ello y que viene al caso es la así llamada dialéctica del amo y del esclavo. Pero la historicidad de la 
historia en el interior de la Fenomenología tampoco es una cosa sencilla. Hay una historia propia de la conciencia hasta el Espíritu, otra del texto mismo (la temporalidad de su dialéctica interna) y otra que sí contiene paralelismos con figuras de la así llamada historia del mundo, o historia en sentido corriente, es decir, figuras históricas reales de la política, religión, filosofía, literatura, etc. ${ }^{1}$

La idea central de estas líneas es entonces poder abocarse a lo que pasa en el interior de la Fenomenología, un texto con sus particularidades históricas y genéticas (de su configuración) propias. Entre la bibliografía actual que se puede encontrar sobre el tema que nos ocupa, destaca sobre todo los artículos del volumen Sterben und Tod bei Hegel (Engelhardt, Neuser, \& Lenski, 2015), y de los cuales es ciertamente el texto de Claus Arthur Scheier el que debemos tener en cuenta, por tratarse justamente de modo exclusivo de la Fenomenología La referencia a este artículo es simplemente para poner en contraposición la lectura transversal de la muerte que quiero presentar, a diferencia de una lectura vertical como hace Scheier. ${ }^{2}$

La lectura "transversal" a la que me refería busca respetar el universo textual de la Fenomenología pero al mismo tiempo evitar hacer una lectura "monotemática" de la muerte, para poder mostrarla en la simpleza de la profundidad del análisis hegeliano. Según ello la figura de la muerte se tematizaría de tres modos diferentes: a) la muerte como la culminación natural de la vida; b) el miedo a la muerte como idea regulativa en el proceso de constitución de una sociedad; c) la superación de la muerte mediante un modo racional-especulativo de sobrevivencia.

Estos tres puntos estructuran sistemáticamente a este artículo. Cada una de estos grupos de temáticas en torno a la muerte presentan particulares dificultades provenientes del horizonte de comprensión de la filosofía de Hegel que debe alcanzarse, para comprender la fuerza metafórica de la idea de la muerte en cada caso. La exposición de los elementos hermenéuticos necesarios se realizará de un modo adaptado al tratamiento de la muerte en cada una de las secciones.

Antes de continuar es fundamental poner en contexto el texto ya que es uno de los pocos textos publicados explícitamente por Hegel y porque la falta

\footnotetext{
${ }^{1}$ Para un desarrollo más amplio de esto, cfr. (Duplancic, 2009)

${ }^{2}$ Scheier toma como figura arquitectónica/metódica principal de la Fenomenología al momento de la reflexión: posición $(\mathrm{P})$, negación $(\mathrm{N})$, negación de la negación $(\mathrm{NN})$ son realizados mediante tres repeticiones en una totalidad de la reflexión. De ello resultaría para él doce momentos a los que corresponden doce figuras de la muerte. La muerte aparece así en una lectura arquitectónica que llamo vertical en relación a la sistematización ascendente que presenta el autor. (Scheier, 2015, pp. 45-46)
} 
de contexto puede ser fatal al momento de hacer una interpretación como de las tantas que uno encuentra sobre la filosofía de Hegel, carentes totalmente de una relación con el pensamiento del autor. Por ello dedicaremos unas muy breves líneas a una introducción a la idea central de la Fenomenología.

\section{La "Idea" de la Fenomenología del espíritu}

La Fenomenología del Espíritu es una de las obras que más polémica ha generado en torno a su idea.

Otto Pöggeler traía a colación ya hace unos años la historia de la problemática de la determinación de la "idea" de la Fenomenología (Pöggeler, 1993). La primera indicación es dada por el mismo Hegel, que en carta a Schelling le comenta poco después de la publicación de la Fenomenología ( $1^{\circ}$ de mayo del 1807) que está expectante de su opinión acerca de la primera parte del sistema que debía ser esa obra. Inmediatamente Hegel agrega que esa primera parte es más bien en realidad "la introducción" al sistema (Hoffmeister, 1961, p. 161). Schelling no contesta esa pregunta. No puede hacerlo porque con la Fenomenología Hegel ha iniciado su propio camino filosófico, que no sólo no puede ser más comprendido desde las premisas de la filosofía de la identidad, sino que ese camino presenta una nueva concepción de cómo debe ser comprendida la filosofía y la historia.

Hegel dice en esa carta que la Fenomenología es la primera parte de su sistema (tal como lo concebía él en 1806), pero que "propiamente" sería la introducción al mismo. Junto a esta aparente contradicción, ingresan otros problemas con los cuales la investigación sobre Hegel se ha enfrentado en los casi doscientos años posteriores a la publicación de la Fenomenología Ellos son, entre otros: el segundo título de la obra (puesto por Hegel en el momento de la impresión del mismo y que fue el definitivo): I. Ciencia de la Fenomenología del espíritu. El primer título rezaba: Primera parte. Ciencia de la experiencia de la conciencia. Como debido a un error de impresión en algunos ejemplares quedó un título, en otros el otro, y en otros ambos, surgieron a partir de allí una serie de interpretaciones que partían de un error básicamente casual ${ }^{3}$. Otros elementos que en el caso de la Fenomenología han "desorientado" a los intérpretes, es la referencia por parte de Hegel a poetas, pensadores y figuras históricas (Siep, 2000, p. 10); la "contradicción" del proyecto de la obra presentado en la Introducción y el finalmente concluido del Prólogo; la diversa estructuración de la obra presentada en el índice y en

\footnotetext{
${ }^{3}$ Para esta temática, cfr: (Hegel, 1988, p. 468; Nicolin, 1967)
} 
el cuerpo del texto; la dificultad para una comprensión global de la obra por el trabajo minucioso de los detalles de algunas partes; y la falta de "forma" en la redacción de las últimas partes (Hoffmeister, 1961, p. 161). Esta serie de dificultades ha llevado a lo que incluso para Pöggeler es un "camino equivocado de más de doscientos años en la interpretación” (Pöggeler, 1993), Vorspann.

Si durante siglos las interpretaciones de estudiantes de Hegel, amigos, discípulos y filósofos han caminado senderos "equivocados", cabe preguntarse con qué derecho se juzga así a una tradición que ya forma parte de la historia de la filosofía. O dicho de otro modo: ¿cuáles son los criterios para la "correcta" interpretación de una obra? ¿existen en realidad algo así como cánones? ¿Y si así es, donde va a parar la libertad en la interpretación, alma de la filosofía? La investigación actual sobre la obra de Hegel se mueve entre estas dos coordenadas: rigor filológico y libertad filosófica. Determinar los límites o espacios "de frontera" de ambos es una tarea en la que estamos en nuestro tiempo. Los trabajos de la Hermenéutica, de la filosofía del lenguaje y de la moderna filología no bastan por sí sólo para una interpretación, si bien no necesariamente "correcta", por lo menos "segura" de la obra hegeliana. A esta seguridad en la interpretación, - o lo que ha sido llamado también, el descubrimiento del verdadero Hegel - es a lo que quería referirse Pöggeler. Ahora bien, su crítica no está dada por la subjetivización de las interpretaciones, sino por lo que puede parecer curioso en un primer momento: por la mala edición de las obras (Pöggeler, 1993, p. 172). Puede parecer curioso digo, porque la Fenomenología por ejemplo, es justamente una obra que ha sido editada por el mismo Hegel. Sin embargo, incluso esta obra, no ha estado ajena de manipulaciones en su edición, que han llevado a la interpretación a caminos equivocados. Y es que estos elementos, en alguna medida "externos" a la filosofía expuesta en el texto, juegan un rol determinante en lo que es la obra de un filósofo. Me refiero a los análisis históricos-filológicos, a los cuales no sólo pertenecen la historia del desarrollo (Entwicklungsgeschichte) de las ideas, sino también las relaciones de esas ideas con la concepción del mundo y de la cultura que les fueron contemporáneas (Geistesgeschichte) y las repercusiones que tuvieron en su tiempo y a posteriori (Wirkungsgeschichte) (Albizu, 1999, p. 34).

Ahora bien, si tomamos el título final de la obra, nos podemos preguntar con Pöggeler:

¿de qué trata propiamente la Fenomenología de la autoconciencia? ¿se trata de investigaciones histórico-religiosas o sobre las tipologías de las concepciones del mundo que se concentran en el concepto de la conciencia desgraciada? 
¿se trata de una historia social bajo la dialéctica del amo y del esclavo, o de una crítica a la sociedad? ¿se trata de preguntas antropológicas y meta-antropológicas por la relación entre la vida y la autoconciencia? o acaso las determinaciones de la lógica son revestidas con el ropaje de las figuras de la conciencia, y si es así, ¿cuáles determinaciones? Si bien la Fenomenología de la autoconciencia puede ser leída de este o aquel modo, parece que la Fenomenología difícilmente se identifica con aquello que Hegel afirmó que era: juna introducción en su filosofía! (Pöggeler, 1993, p. 235)

Introducción a su filosofía significaba por aquel entonces, introducción a un sistema filosófico, un sistema superador del de Schelling y claramente del de Kant. En este sentido la Fenomenología debería ser el camino que hace la conciencia en tanto pasa de un saber aparente o saber inmediato, a un saber reflexionado, que es un saber con niveles incrementantes de mismidad, pues en este saber de si sabe de su objeto, como siendo en sí y también para sí.

Introducción a la filosofía de Hegel significaba también para él una introducción que llevara a las puertas del saber puro, de un saber sin mediación exterior, un saber absoluto. La introducción debiera ser el camino del saber relativo al saber absoluto. De allí la referencia cruzada de la introducción de la Lógica de 1812.

Introducción en tanto experiencia de la conciencia es comprender que la experiencia nunca se trata de salir del pensar, sino del movimiento por el cual lo otro determina al pensar y el pensar determina a lo otro. La experiencia es por lo tanto el desarrollo genético de las posibilidades de la conciencia respeto a lo otro que ella, desarrollo que se descubre en la unidad de su diferencia y deviene autoconciencia, autoconciencia que se descubre siendo toda realidad y deviene razón. Esta razón que es toda realidad, es por qué no decirlo, otra razón que la razón pura, es Espiritu. (Duplancic, 2014)

En este camino de la experiencia de la conciencia, del saber aparente al saber real y absoluto, de la conciencia al Espíritu el ritmo del caminar es con del ductus de la dialéctica o escepticismo que se consuma. En ese escepticismo que no se detiene en un momento solamente del negar -momento de la alienación (Duplancic, 2008)- se produce el negar la negatividad, y como tal se producen momentos del morir. Estos momentos se dan por supuesto en aquellos casos en que algo pueda morir, es decir, que haya organicidad para que se pueda producir un paso del mundo de la naturaleza al mundo del espíritu. Lo negativo es así entendido como un momento de lo ideal, absoluto, divino, en contraposición con lo finito, natural, no ideal. (Wandschneider, 2015, p. 55) 


\section{El simple morir}

En este punto se considera a la muerte como simple culminación natural de la vida, un significado de la muerte que para Hegel es el más pobre dentro del horizonte especulativo de la Fenomenología. En un primer momento encontramos a esta significación de la muerte en la "lucha a vida o muerte" del capítulo de la autoconsciencia. En el desprecio de la muerte por parte de una de las autoconsciencias se ve la confirmación efectiva del señorío sobre la vida (sobre el ser). Pero la muerte en tanto tal representa otro aspecto fundamental que se encuentra en el horizonte en el que se desenvuelve la mencionada dialéctica de la lucha. La muerte es la culminación de la movilidad del concepto en tanto mediante ella las autoconsciencias (en cuanto autoconsciencias muertas) dejan de ser extremos (Hegel, 1988, p. 112) que se refieren mutuamente en una relación expresamente querida y, en cuanto tal, configurante del movimiento del reconocimiento. Ahora bien, esta muerte, como "negación natural" de la vida, una "negación sin mismidad", no trae otra cosa que la esclavitud de la necesidad. Esta esclavitud es la dependencia de la vida y del ser. Ella es -en un primer momento- la "cadena" del esclavo (Gadamer, 1998, p. 241). Este aspecto será profundizado en el capítulo V (razón), sección B, Apartado a. "El placer y la necesidad". En este lugar la muerte es caracterizada como la "necesidad sin vida" (Hegel, 1988, p. 201). En ello se ve la lógica inherente al placer (Lust), el que como trasgresión de la ley -ley que toma la forma de la fuerza destructiva y castigadora de la sociedad (Hegel, 1988, p. 200) - toma la vida para sí (en cuanto la goza hasta el final), pero con ello -y justamente por ello- se encuentra más bien con la muerte (Hegel, 1988, p. 201). Este referencia hegeliana al Fausto de Goethe es aplicable a su vez -como ha señalado Hyppolite o Siep- también a la experiencia humana del ver culminar la vida, en el sentido de que nuestra singularidad se va agotando con cada momento de placer y con ello vamos muriendo de a poco, nos vamos "usando" y acabando (Hyppolite, 1991, p. 255; Siep, 2000, pp. 150-151). Ahora bien, este momento fáustico sobre la vida no indica solamente al hecho de que finalmente sólo se encuentra la muerte y la necesidad de la nada. También se ve allí -como lo hace Siep- la figura del placer como un permanente destruir límites y fronteras. Una figura que -como es visible en la tradición filosófica desde el Filebo de Platón- encara contra el principio de la individualidad y de la libertad en cuanto independencia. Porque individualidad es auto-limitarse frente a otro y la carencia de límites del placer contradice justamente a ello. Pero también contradice la otra forma de la individualidad que se alcanza en la figura del amor mediante la fusión con el otro -como Hegel lo ha insinuado con la referencia a las figu- 
ras de Fausto y Gretchen. De este modo resulta problemático querer interpretar a toda la Fenomenología desde la perspectiva de un motivo fáustico. Las formas de la emancipación y rebelión no son inmediatamente identificables con una auto-liberación de la enajenación que llevaría a cabo la humanidad -como quiere ver Bloch (Bloch, 1961, pp. 170-171). Estas formas representarían más bien formas imperfectas y enajenadas de la libertad, en cuanto estas no son superadas y elevadas a la libertad del espíritu.

Otro ejemplo de ello es una forma igualmente improductiva de la muerte como es la de la "libertad absoluta". Allí la muerte es "la única obra" y el único "hacer" de esa forma de libertad. Hegel la describe como:

una muerte que no tiene ni contenido ni plenitud, pues lo que es negado es el punto igualmente vacío del sí mismo libre-absolutista. Es la muerte más fría, una mera muerte, sin más significación que la de cortarle a alguien la cabeza o el tomar un vaso de agua (Hegel, 1988, p. 320).

\section{La muerte como factor socializante}

A lo largo de la Fenomenología la muerte no se presenta solamente como la culminación natural de la vida. La muerte juega además un rol regulativo (por ejemplo como "terror") para la constitución de la sociedad. Ese rol de la muerte puede ser visto a partir de la función que toma la idea de muerte para "sacudir" todas las fijaciones que posee la conciencia dentro del camino de la Fenomenología. Estas "fijaciones" pertenecen a un modelo de racionalidad que no ve la movilidad del concepto y por ello permanece encerrada en una estructura conceptual estática. Esa función de la muerte la describe Hegel como "el amo absoluto" (Hegel, 1988, pp. 114, 246, 321) o "lo más terrible" (Hegel, 1988, p. 27). Este "sacudón" que produce la muerte se ve en la figura del "siervo" de una manera ejemplar. Hegel dice:

La conciencia no ha tenido miedo ante esto $\mathrm{u}$ aquello ni tampoco frente a este $u$ otro momento, sino que ha temido en su esencia misma, porque ella ha sentido el temor frente a la muerte, el señor absoluto. (Hegel, 1988, p. 114).

En el caso de la figura del siervo, la muerte contribuye a la formación de la conciencia en el marco de subjetividades aisladas. La muerte como "el señor absoluto" o "temor" también se encuentra en otros pasajes pero en estos otros casos ella juega un rol específico en el marco de relaciones ínter-subjetivas (dentro de la eticidad). La muerte se presenta acá como temor y ese temor es utilizado en contra de la singularidad de subjetividades aisladas, de modo que 
éstas se adecuen a los intereses y leyes de lo colectivo. El primer pasaje con esta significación se puede encontrar en el capítulo VI, dentro de la sección "A. El espíritu verdadero, la eticidad". Hegel tematiza acá el momento del ser comunitario y describe la función del Gobierno como defensora del ser común frente a la amenaza de los "sistemas que se aíslan a sí mismo". Con ello se refiere a las singularidades personales, a la propiedad, al derecho personal, etc.). Hegel dice:

El espíritu del llegar a ser juntos (Zusammenkunft) es la simplicidad y la esencia negativa de esos sistemas que se aíslan a sí mismos. Y para no quedar fijo y enraizado en ese aislamiento (y mediante ello dejar caer el todo y volar al espíritu), el gobierno debe sacudir de tiempo en tiempo a esos sistemas en su interior mediante guerras, confundiendo e hiriendo el orden y derecho de la independencia de que gozan. Y mediante ello podrá hacer sentir a los individuos que buscan separarse del todo y refugiarse en el ser para sí intangible y en la seguridad de la persona jurídica, obligados a entregarse al trabajo del servicio de su señor, la muerte. (Hegel, 1988, p. 246).

Esta función, que aparentemente se dirige contra las libertades individuales tiene, empero, para Hegel, una función liberadora:

El espíritu se separa así mediante esa disolución de la permanencia del hundimiento en el ser que se produce en el plano de la eticidad. Y contiene y eleva al sí mismo de la conciencia hacia la libertad y hacia su fuerza. (Hegel, 1988, p. 246)

Una función similar de la muerte con este carácter represivo se puede encontrar más adelante en la sección $\mathrm{B}$ de ese mismo capítulo, cuando se presenta a la libertad absoluta de la revolución francesa. Aquí la muerte juega su rol como temor en el trabajo de disciplinamiento y organización de los individuos en "masas espirituales" (Hegel, 1988, p. 321). La guerra como instrumento para integrar a los ciudadanos en la eticidad es -como señala Siep- un viejo pensamiento no sólo de la filosofía (Platón, Kant), sino de Hegel mismo. Éste había relacionado en su escrito La constitución alemana a la caída del reino antiguo con los movimientos revolucionarios basados en los intereses individuales y en las relaciones jurídicas privadas entre Individuos o entre estamentos y estado (Siep, 2000, p. 182). 


\section{El significado especulativo de la muerte}

El tercer significado de la muerte en la Fenomenología según la lectura propuesta, es el especulativo -o la superación del temor. Al comienzo de la Fenomenología, en la introducción, Hegel habla acerca del fin de la obra y dice:

La progresión hacia... la meta es... incontenible, y no puede encontrar satisfacción en ninguna estación anterior. Lo que se limita a una vida natural no puede por sí mismo ir más allá de su existencia inmediata, sino que es empujado más allá por otro, y este ser arrancado de su sitio es su muerte. (Hegel, 1988, p. 57)

Esta progresión se da mediante la ascendente presencia del sí mismo (como negación determinada) en cada nivel de la experiencia. El agotamiento de posibilidades de significación en cada uno de esos niveles se presenta para cada uno de los estadios de la Fenomenología como su muerte. Este agotamiento de la significancia para la conciencia que hace la experiencia, es para nosotros la incapacidad de aquella de ver y aceptar la presencia del sí-mismo, o dicho de otro modo, de aceptar la positividad siempre nueva que surge de la negación determinada. La salida especulativa de este agotamiento de la significancia por parte de la conciencia consiste para Hegel en mirar a la muerte, a lo más temible, a los ojos, y allí descubrir la fuerza de la vida del concepto. Esto significa: dejar operar la negación determinada, entregarse a la fuerza del escepticismo que se consuma. Ahora bien, esta actitud de aceptación de la negación determinada, que es un modo de "ir más allá" de la muerte, ¿qué rol juega en las relaciones intersubjetivas antes aludidas? En un primer momento se puede decir que de lo que se trata es de "sobrevivir" la muerte natural o de una forma de temporalidad-del concepto- que sea más "larga" que las formas de la vida para la conciencia natural. Este sería un ser más extensivo que el simple mirar momentáneo de la conciencia.

Esta forma de muerte "especulativa" o la superación de la muerte natural que se menciona en la introducción, vuelve a surgir con este carácter conceptual en el capítulo IV, sección "A", en la consideración de la lucha a vida o muerte y, lo que sigue a ello, en de la llamada "dialéctica del amo y del siervo". En ese contexto se busca la muerte del otro para verificar la propia autonomía. Porque esto se da en una primera forma (la más primitiva por decirlo así) del espíritu, intentar matar a otro significa aquí, automáticamente poner en riesgo la propia vida, pues la otra autoconsciencia busca el mismo reconocimiento que la primera. Como se sabe, la simple muerte de una de las autoconsciencias lleva consigo a la tumba toda posibilidad de reconocimien- 
to. Pero sobrevivir simplemente, tampoco es una solución. Hegel introduce aquí su solución especulativa. Esta consiste en experimentar la muerte, en tanto la autoconsciencia llega a ser consciente del significado de la muerte y de la vida, esto es, en tanto y cuanto la autoconsciencia se reconoce como un momento pleno de negatividad y así de vida espiritual.

Esta afirmación del sí mismo (Hegel habla también de ser para sí) sobre la muerte se encuentra nuevamente en el capítulo VI, sección "A", apartado “a. El mundo ético...". Aquí introduce Hegel esta función especulativa del sí mismo en un mundo ético. Esto se presenta en la medida en que se señala la obligación de los parientes de un muerto de otorgarle a éste nuevamente una autoconsciencia, de modo que no pertenezca más a la naturaleza solamente y permanezca de otro modo como un ser pasivo o una individualidad vacía. Mediante el rito y las obligaciones para con el muerto se va más allá de los límites del tiempo real y se lo introduce en el tiempo espiritual de la eticidad. Esta obra de los familiares consiste en llevar el muerto en cuanto "ser natural devenido inmediato" hacia un ser espiritual mediado. De este modo se le otorga al muerto un modo de supervivencia espiritual ${ }^{4}$.

Otra posibilidad de la supervivencia sería la que arriba se mencionó al pasar, a saber, el hecho de sobrevivir a la lucha a vida y muerte. Esta posibilidad que Hegel no considera en el capítulo de la autoconsciencia, se puede ver en el capítulo VI, en la exposición de la "Formación y su reino de la realidad". El que sobrevive a la lucha como vencedor se convierte en este caso en peligroso, pues el polo contrario del reconocimiento está muerto. Esto significa que el que ha sobrevivido ha sido reconocido hasta la muerte, en su máxima expresión. Y por ello no necesita más ser reconocido. Esta afirmación unilateral del individual es nombrada por Hegel como la conciencia vil. Hegel dice:

El sacrificio del existente (Dasein) que se lleva a cabo en el servicio, sólo es completo cuando llega hasta la muerte; pero el peligro de la muerte misma, cuando es superado y se sobrevive a él, deja en pie un determinado existente $\mathrm{y}$, por tanto, un para sí particular, que hace ambiguo y sospechoso el consejo en pro del bien universal y que, de hecho, se reserva la propia suposición y la voluntad particular frente al poder del Estado. Este existente sigue, pues, comportándose de un modo desigual con respecto al poder del Estado y cae bajo la determinación de la conciencia vil, consistente en que está siempre dispuesta a sublevarse. (Hegel, 1988, p. 275)

\footnotetext{
${ }^{4}$ Hegel alude todo el tiempo en este caso al comportamiento de Antígona. Cfr. por ejemplo: (Seibold, 1993 , p. 53 ss.)
} 
La única salida para este dilema será para Hegel naturalmente especulativa, pero con formas reales de acción. Formulado con sus palabras, la particularidad de la conciencia deberá ser sacrificada (Hegel, 1988, pp. 207-208). Sólo de este modo se podrá sobrevivir la muerte y no ser un simple muerto:

El verdadero sacrificio del ser para sí sólo es, por tanto, aquel en que se entrega, de un modo tan total como en la muerte, pero manteniéndose igualmente en esta enajenación; el ser para sí deviene con ello real, como lo que es en sí, como la unidad idéntica de sí mismo y de sí como lo contrapuesto. (Hegel, 1988, p. 275)

Esta posibilidad del "morir" y del "vivir" especulativos ejercerá su rol decisivo en el capítulo "Religión". La muerte es en primer lugar la consecuencia de las guerras entre los pueblos, que en servicio de sus dioses (con forma animal en este caso) luchan (Hegel, 1988, p. 371). Mediante ello ingresaría la contradicción y la negatividad excluyente en lo divino mismo (Siep, 2000, p. 226). Este significado especulativo de la muerte gana su rol central en la historia de la religión, que para Hegel no es otra cosa que la historia de la encarnación de Jesucristo. Mediante la encarnación y la muerte del hijo de Dios se realiza el devenir de la autoconsciencia individual hacia la autoconsciencia universal o hacia la comunidad. Esto es, la muerte pasará de tener el significado del no-ser del individuo (como se expuso en el primer apartado) a arribar a la universalidad del espíritu, que vive en su comunidad y que allí muere y resucita diariamente (Hegel, 1988, p. 418). La superación especulativa de la muerte será presentada al final de la obra (en su prólogo) en su forma lógica, a saber, como vida del espíritu: una vida que no se avergüenza frente a la muerte, sino que la soporta y la contiene. El espíritu alcanza de este modo su vida -la meta de la Fenomenología- en tanto él mismo se encuentra en este desgarramiento absoluto (Hegel, 1988, p. 27). Este aspecto del ser-cabe-sí del espíritu en su desgarramiento (muerte) no es otra que la libertad especulativa que como absolutez del absoluto construye la intersubjetividad (Hegel, 1988, p. 108).

\section{Conclusión}

A lo largo del camino recorrido hemos podido observar cómo se muestra la figura de la muerte de la mano de la dialéctica propia de la Fenomenología, esto es, de modos incrementantes de la experiencia en cuanto desarrollo genético de las posibilidades de la conciencia respecto a lo otro que ella. Lo que entonces podríamos llamar la "experiencia de muerte" ha sido presentada en 
el marco de una lectura centrada en los movimientos lógicos inherentes a la obra. La interpretación se ha hecho asimismo teniendo en cuenta el contexto de su génesis propia más que las contextualizaciones historiográficas. En esta lectura vemos cómo la muerte se presenta bajo diversas figuras. Estas figuras se ordenan en un camino que va de la conciencia natural al espíritu absoluto; y en cuanto figuras del desarrollo muestran sus "insuficiencias" parciales respecto a la figura de la muerte como último eslabón especulativo

\section{Referencias bibliográficas}

Albizu, Edgardo (1999). Tiempo y Saber absoluto. La condición del discurso metafisico en la obra de Hegel. Buenos Aires: Jorge Baudino Ediciones, UNSAM.

Bataille, Georges, \& Strauss, Jonathan (1990). Hegel, Death and Sacrifice. In: Yale French Studies (78), pp. 9-28. doi:10.2307/2930112

Bloch, Ernst (1961). Das Faustmotiv der Phänomenologie des Geistes. In: Hegel Studien, 1, pp. 155-171.

Duplancic, Victor (2008). De la Methexis a la alienación: o el reverso de la libertad. In: Philosophia, pp. 81-102.

Duplancic, Victor (2009). Tres temporalidades en el interior de la Fenomenología del Espíritu. In: Revista Filosofía UIS, 6 (1-2), pp. 45-56.

Duplancic, Victor (2014). La Fenomenología del Espíritu como punto de inflexión filosófica de Hegel. In H. Ferreiro, T. S. Hoffmann, \& A. Bavaresco (Eds.), Los aportes del itinerario actual de Kant a Hegel (pp. 467-491). Buenos Aires: Editora Fi: EDIPUCRS.

Engelhardt, Dietrich von, Neuser, Wolfgang, \& Lenski, Wolfgang (Eds.) (2015). Sterben und Tod bei Hegel. Würzburg: Königshausen \& Neumann.

Gadamer, Hans-Georg (1998). Hegels Dialektik des Selbstbewusstseins. In H. F. Fulda \& D. Henrich (Eds.), Materialien zu Hegels „,Phänomenologie des Geistes “ (pp. 217-242). Frankfurt am Main: Suhrkamp.

Gemerchak, Christopher M. (2012) Sunday of the Negative, The: Reading Bataille Reading Hegel: SUNY Press.

Hegel, Georg Wilhelm Friedrich (1988). Phänomenologie des Geistes (W. Bonsiepen \& R. Heede Eds. Vol. 9). Hamburg: Meiner. 
Hoffmeister, J. (Ed.) (1961). Briefe von und an Hegel (Vol. I: 1785-1812). Hamburg: Meiner.

Hyppolite, Jean (1991). Génesis y estructura de la Fenomenología del espiritu de Hegel (Vol. 105). Barcelona: Península.

Kojève, Alexandre (1947). Introduction à la lecture de Hegel. Leçons sur la Phénoménologie de l'Esprit professées de 1933 à 1939 à l'Ecole des Hautes-Etudes. Paris.

Kojève, Alexandre (1972). La dialéctica de lo real y la idea de la muerte en Hegel. Buenos Aires: La Pleyade.

Nicolin, Friedrich (1967). Zum Titelproblem der Phänomenologie des Geistes. In: Hegel Studien, 4.

Pöggeler, Otto (1993). Hegels Idee einer Phänomenologie des Geistes (2. ed.). Freiburg, München: Alber.

Scheier, Claus-Artur (2015). Gestalten des Todes in Hegels "Phänomenologie des Geistes". In D. von Engelhardt, W. Lenski, \& W. Neuser (Eds.), Sterben und Tod bei Hegel (pp. 43-54). Würzburg: Königshausen \& Neumann.

Seibold, Jorge R. (1993). Pueblo y saber en la fenomenología del espiritu de Hegel. Buenos Aires: Ediciones Universitarias del Salvador y Diego de Torres.

Siep, Ludwig (2000). Der Weg der Phänomenologie des Geistes. Ein einführender Kommentar zu Hegels Differenzschrift und Phänomenologie des Geistes. Frankfurt am Main: Suhrkamp.

Wandschneider, Dieter (2015). 'Schmerz der Negativität' und Tod in Hegels Konzeption des Geistes. In D. v. Engelhardt, W. Neuser, \& W. Lenski (Eds.), Sterben und Tod bei Hegel (pp. 55-68). Würzburg: Königshausen \& Neumann. 